Original Contributions

\title{
Molecular mechanism of metal-independent decomposition of lipid hydroperoxide 13-HPODE by halogenated quinoid carcinogens
}

\author{
Hao Qin ${ }^{\text {a }}$, Chun-Hua Huang ${ }^{\text {a }}$, Li Mao ${ }^{a}$, Hai-Ying Xia ${ }^{a}$, Balaraman Kalyanaraman ${ }^{\text {b }}$ \\ Jie Shao ${ }^{a}$, Guo-Qiang Shan ${ }^{\text {a }}$, Ben-Zhan Zhu ${ }^{\text {a,c,* }}$ \\ a State Key Laboratory of Environmental Chemistry and Ecotoxicology, Research Center for Eco-environmental Sciences, Chinese Academy of Sciences, \\ Beijing 100085, China \\ ${ }^{\mathrm{b}}$ Department of Biophysics, Medical College of Wisconsin, Milwaukee, WI 53226, USA \\ ${ }^{\mathrm{c}}$ Linus Pauling Institute, Oregon State University, Corvallis, OR 97331, USA
}

\section{A R T I C L E I N F O}

\section{Article history:}

Received 22 January 2013

Received in revised form

2 May 2013

Accepted 3 May 2013

Available online 14 May 2013

\section{Keywords:}

Halogenated quinones

13-Hydroperoxy-9,11-octadecadienoic acid

ESR spin trapping

Lipid alkyl radicals

4-Hydroxy-2-nonenal

Free radicals

\begin{abstract}
A B S T R A C T
Halogenated quinones are a class of carcinogenic intermediates and newly identified chlorination disinfection by-products in drinking water. 13-Hydroperoxy-9,11-octadecadienoic acid (13-HPODE) is the most extensively studied endogenous lipid hydroperoxide. Although it is well known that the decomposition of 13-HPODE can be catalyzed by transition metal ions, it is not clear whether halogenated quinones could enhance its decomposition independent of metal ions and, if so, what the unique characteristics and similarities are. Here we show that 2,5-dichloro-1,4-benzoquinone (DCBQ) could markedly enhance the decomposition of 13-HPODE and formation of reactive lipid alkyl radicals such as pentyl and 7-carboxyheptyl radicals, and the genotoxic 4-hydroxy-2-nonenal (HNE), through the complementary application of ESR spin trapping, HPLC-MS, and GC-MS methods. Interestingly, two chloroquinone-lipid alkoxyl conjugates were also detected and identified from the reaction between DCBQ and 13-HPODE. Analogous results were observed with other halogenated quinones. This represents the first report that halogenated quinoid carcinogens can enhance the decomposition of the endogenous lipid hydroperoxide 13-HPODE and formation of reactive lipid alkyl radicals and genotoxic HNE via a novel metal-independent nucleophilic substitution coupled with homolytic decomposition mechanism, which may partly explain their potential genotoxicity and carcinogenicity.
\end{abstract}

(c) 2013 Elsevier Inc. All rights reserved.

\section{Introduction}

Lipid peroxidation (LPO) has been the subject of extensive studies for several decades, and its mechanisms, dynamics, and products are now fairly well established [1-5]. The free radical nature of the chain reaction and the three distinctive stages of the

Abbreviations:: 13-HPODE, 13-hydroperoxy-9,11-octadecadienoic acid; LPO, lipid peroxidation; DCBQ, 2,5-dichloro-1,4-benzoquinone; HNE, 4-hydroxy-2-nonenal; ESR, electron spin resonance; HPLC-MS, high-performance liquid chromatographymass spectrometry; GC-MS, gas chromatography-mass spectrometry; $t$ - $\mathrm{BuOOH}$, $t$-butylhydroperoxide; POBN, $\alpha$-(4-pyridyl-1-oxide)- $N$-tert-butyl nitrone; DMPO 5,5-dimethyl-1-pyrroline $N$-oxide; FTICR-MS, Fourier transform ion cyclotron resonance-mass spectrometry; $\mathrm{CBQ}-\mathrm{OH}, 2$-chloro-5-hydroxy-1,4-benzoquinone; $\mathrm{CBQ}-\mathrm{OO}-t-\mathrm{Bu}, 2$-chloro-5-t-butylperoxyl-1,4-benzoquinone; $\mathrm{CBQ}(\mathrm{OH})-\mathrm{O}-t-\mathrm{Bu}$, 2-hydroxy-3-t-butoxy-5-chloro-1,4-benzoquinone; CBQ-13-OOL,

2-chloro-5-(L-13-OO)-1,4-benzoquinone; CBQ(OH)-13-O-L,

2-hydroxy-3-(L-13-oxy)-5-chloro-1,4-benzoquinone; CBQ(OH)-9-O-LO,

2-hydroxy-3-(OL-9-oxy)-5-chloro-1,4-benzoquinone.

* Corresponding author at: State Key Laboratory of Environmental Chemistry and Ecotoxicology, Research Center for Eco-environmental Sciences, Chinese Academy of Sciences, Beijing 100085, China. Fax: +86 1062923563.

E-mail address: bzhu@rcees.ac.cn (B.-Z. Zhu). process as initiation, propagation, and termination have been well defined [1-3]. Lipid hydroperoxides are formed as the major primary products of propagation that have been liberated from cell membranes by phospholipases and lipoxygenase enzymes [1-5]. 13-Hydroperoxy-9,11-octadecadienoic acid (13-HPODE; also called 13-hydroperoxylinoleic acid) is one of the most extensively studied endogenous lipid hydroperoxides and is generated in vivo from linoleic acid by 15-lipoxygenases [1-5]. The decomposition of lipid hydroperoxides has been extensively studied because, in biological systems, lipid-derived free radicals are known to cause damage to biomembranes, proteins, and other biomolecules. In the presence of transition metal ions, especially ferrous iron $\mathrm{Fe}(\mathrm{II})$, 13-HPODE could first generate lipid alkoxyl radical, which will be decomposed, via $\beta$-scission, cyclization, and spin isomerization, to form reactive lipid alkyl radicals such as pentyl and 7-carboxyheptyl radicals [6,7] (see Supplementary Scheme S1) and several lipid acid products [8] (Supplementary Scheme S2). 13-HPODE could also be decomposed to produce genotoxic aldehydes such as 4-hydroxy-2-nonenal (HNE) as the end products via cleavage of adjacent $\mathrm{C}-\mathrm{C}$ bonds $[1,9]$ (Supplementary Scheme S3), which has been implicated in the pathogenesis of 
various diseases, including atherosclerosis, cancer, and neurodegenerative diseases [1].

We have shown recently that alkoxyl radical and carboncentered quinone ketoxy radical can be produced during the metal-independent decomposition of a model organic hydroperoxide, $t$-butylhydroperoxide $(t-\mathrm{BuOOH})$, by halogenated quinones such as 2,5-dichloro-1,4-benzoquinone (DCBQ) [10,11], which was proposed through the following mechanism: a nucleophilic reaction may take place between $\mathrm{DCBQ}$ and $t-\mathrm{BuOOH}$, forming a quinone-peroxide reaction intermediate, $\mathrm{CBQ}-\mathrm{OO}-t-\mathrm{Bu}$, which can decompose homolytically to produce the alkoxyl radical $t$-BuO ${ }^{\circ}$ and oxygen-centered quinone enoxy radical $\mathrm{CBQ}-\mathrm{O}^{*}$. $\mathrm{CBQ}-\mathrm{O}^{-}$then either disproportionates to form one of the major reaction products, $\mathrm{CBQ}-\mathrm{OH}$, or isomerizes to form the carboncentered quinone ketoxy radical ${ }^{\circ} \mathrm{CBQ}=\mathrm{O}$, which then couples with $t-\mathrm{BuO}^{*}$ to produce another major reaction product, $\mathrm{CBQ}(\mathrm{OH})-$ $\mathrm{O}-t$-Bu, via keto-enol tautomerization (see Scheme 2 in Ref. [7]). We also found that the highly reactive hydroxyl radicals and chemiluminescence could be produced by hydrogen peroxide and halogenated quinones via a similar metal-independent mechanism, which could be inhibited by hydroxamic acids [1216].

Because 13-HPODE is a secondary hydroperoxide that also has a long lipid chain, whereas $t$ - $\mathrm{BuOOH}$ is a tertiary hydroperoxide with only a small $t$-butyl group, we speculated that the reactions between DCBQ and the two organic hydroperoxides (13-HPODE and $t-\mathrm{BuOOH}$ ) should have not only similarities, but also differences. Therefore, in this study, we addressed the following questions: (1) Could DCBQ and other halogenated quinones enhance the decomposition of 13-HPODE and the formation of the reactive radical intermediates and the genotoxic aldehydes independent of transition metal ions? (2) If so, what are the similarities and unique differences (i) between the decomposition of 13-HPODE and that of $t$-BuOOH by DCBQ and (ii) between DCBQ- and ironmediated decomposition of 13-HPODE? (3) What is the underlying molecular mechanism?

\section{Materials and methods}

\section{Chemicals}

DCBQ 2,6-dichloro-1,4-benzoquinone (2,6-DCBQ), tetrachloro1,4-benzoquinone (TCBQ), $\alpha$-(4-pyridyl-1-oxide)- $N$-tert-butyl nitrone (POBN), O-(2,3,4,5,6-pentafluorobenzyl)hydroxylamine (PFBHA), and $\mathrm{N}$-methyl- $\mathrm{N}$-(trimethylsilyl)trifluoroacetamide (MSTFA) were purchased from Sigma-Aldrich.

\section{Preparation of 13-HPODE}

13-HPODE was prepared using linoleic acid (50 mg) and soybean lipoxidase (200,000 U, type $\mathrm{V}$ ) in $30 \mathrm{ml}$ of $0.2 \mathrm{M}$ borate buffer (pH 9.0). The solution was mixed by magnetic stirring while the temperature was kept at $25^{\circ} \mathrm{C}$. After $1 \mathrm{~h}$, the reaction mixture was acidified to $\mathrm{pH} 3$ with $1 \mathrm{~N} \mathrm{HCl}$ and 13-HPODE was extracted with diethyl ether. The combined extracts were washed with water, dried over sodium sulfate, and evaporated under a stream of nitrogen. The 13-[S- $(Z, E)]-H P O D E$ was then purified using a normal-phase system. 13-(E,E)-HPODE had a retention time of 11.9 min under these conditions, and 13-(Z,E)-HPODE had a retention time of $13.2 \mathrm{~min}$. The pure 13-[S-(Z,E)]-HPODE was dissolved in ethanol, and its concentration was determined by UV spectroscopy $\left(\lambda_{\max } 234 \mathrm{~nm}, \varepsilon=25,600 \mathrm{~L} / \mathrm{mol} \mathrm{cm}\right)$. The stock solution of $13-\mathrm{HPODE}$ was stored at $-70^{\circ} \mathrm{C}$.
Synthesis of 5-chloro-2-hydroxy-1,4-benzoquinone (CBQ-OH)

$\mathrm{CBQ}-\mathrm{OH}$ was synthesized as described in our previous publications $[10,11]$.

\section{ESR spin trapping studies}

The basic system used in this study consisted of DCBQ dissolved in dimethyl formamide (DMF; final DMF concentration, 1\%), 13-HPODE dissolved in ethanol (final ethanol concentration, 10\%), and the spin trapping agent POBN $(20 \mathrm{mM})$, in Chelex-treated phosphate buffer (100 mM, pH 7.4) at room temperature. ESR spectra were recorded $1 \mathrm{~min}$ after the interaction between 13-HPODE and DCBQ at room temperature under normal room-lighting conditions on a Bruker ER 200 D-SRC spectrometer operating at $9.8 \mathrm{GHz}$ and a cavity equipped with a Bruker Aquax liquid sample cell. Typical spectrometer parameters were as follows: scan range, $100 \mathrm{G}$; field set, $3405 \mathrm{G}$; time constant, $200 \mathrm{~ms}$; scan time, $100 \mathrm{~s}$; modulation amplitude, $0.25 \mathrm{G}$; modulation frequency, $100 \mathrm{kHz}$; receiver gain, $1.25 \times 10^{5}$; and microwave power, $20 \mathrm{~mW}$.

\section{Mass spectral studies}

Electrospray ionization triple-quadrupole mass spectrometry (Quattro Premier XE; Waters) was used to detect and identify POBN adducts. MS and MS/MS spectra were acquired in positive-ion mode. The operating parameters were capillary and sample cone voltages, $3 \mathrm{kV}$ and $30 \mathrm{~V}$; source and desolvation temperatures, 80 and $200{ }^{\circ} \mathrm{C}$; nitrogen used as the drying gas; argon used as the collision gas at the pressure of $5.0 \times 10^{-5}$ Torr $(1 \mathrm{Torr}=133.322 \mathrm{~Pa})$, and collision energy, $15 \mathrm{~V}$. Because of the low levels of spin trapped radical products in the reaction mixture, the selective reaction monitoring (SRM) mode of the calculated protonated molecule mass $( \pm 0.5 \mathrm{Da}$ ) of predicted products was used. The chosen transitions were $m / z$ 266/179 for POBN/pentyl radical adducts and $m / z$ 338/251 for POBN/7-carboxyheptyl radical adducts.

The final reaction products of the reaction between DCBQ and 13-HPODE were analyzed by electrospray ionization quadrupole time-of-flight (TOF) mass spectrometry (Q-Tof Micro; Waters). MS and MS/MS spectra were acquired in negative-ion mode. Capillary and sample cone voltages were $2.5 \mathrm{kV}$ and $30 \mathrm{~V}$, source and desolvation temperatures were 80 and $200{ }^{\circ} \mathrm{C}$. Nitrogen was used as the drying gas. The collision gas was argon at the pressure of $5.0 \times 10^{-5} \mathrm{Torr}$, and the collision energy was $10 \mathrm{~V}$. Both the high and the low resolution for mass filter were set at $5.0 \mathrm{~V}$. The pressure in the TOF cell was lower than $3.0 \times 10^{-7}$ Torr. Full-scan spectra were recorded in profile mode. The range between $\mathrm{m} / \mathrm{z} 100$ and 600 was recorded at a resolution of 5000 (FWHM), and the accumulation time was $1 \mathrm{~s}$ per spectrum. For MS/MS studies, the quadrupole was used to select the parent ions, which were subsequently fragmented in a hexapole collision cell by using argon as the collision gas and an appropriate collision energy (typically 10-20 eV).

Data acquisition and analysis were carried out by using MassLynx software (Waters; version 4.0).

\section{HPLC conditions}

Normal-phase chromatography was used to purify 13-HPODE. It was performed using an Agilent 1200 HPLC system, with a Venusil XBP-Si column $(25 \mathrm{~cm} \times 10 \mathrm{~mm}, 5 \mu \mathrm{m}$; Agela $)$ at a flow rate of $4.7 \mathrm{ml} / \mathrm{min}$. Isocratic elution was performed with hexane and 2-propanol (197:3, v/v) containing 0.1\% acetic acid, and the UV detector was set at $234 \mathrm{~nm}$ for detection of conjugated dienes.

The intermediate and final products between DCBQ and 13-HPODE were analyzed with an HPLC apparatus (Waters 2695 XE) 
including an Eclipse Plus C18 column $(15 \mathrm{~cm} \times 4.6 \mathrm{~mm}, 5 \mu \mathrm{m}$; Agilent). Gradient elution was performed in the linear mode. Two solvents were used: solvent $A$ was $0.1 \%$ acetic acid and solvent B was acetonitrile. The gradient conditions were as follows: isocratic elution with $10 \% \mathrm{~B}$ was conducted for $10 \mathrm{~min}$ at a flow-rate of $1 \mathrm{ml} / \mathrm{min}$. A linear gradient was then run to $90 \% \mathrm{~B}$ for $30 \mathrm{~min}$ at a flow rate of $1 \mathrm{ml} / \mathrm{min}$.

\section{The derivatization of aldehyde products}

For the pentafluorobenzyl (PFB) derivatization of aldehydes, the reaction products $(200 \mu \mathrm{l})$ were mixed with a $50 \mathrm{mM}$ PFBHA methanolic solution $(100 \mu \mathrm{l})$ and stored at room temperature for $2 \mathrm{~h}$. The PFB-oxime derivatives were extracted twice with $500 \mu \mathrm{l}$ of $n$-hexane, and the organic layers were dried under $\mathrm{N}_{2}$ gas and in a vacuum desiccator. For the trimethylsilyl (TMS) derivatization of hydroxyl groups in the PFB-oximes, $50 \mu \mathrm{l}$ of MSTFA was added to the samples and incubated at room temperature for $1 \mathrm{~h}$.

\section{GC-MS analysis of HNE}

GC-MS was performed using a $6890 \mathrm{~A}$ mass spectrometer (Agilent) equipped with an HP-5 fused silica capillary column (30 $\mathrm{m} \times 0.25 \mathrm{~mm}, 10 \mu \mathrm{m}$ film thickness; Supelco, Bellefonte, PA, USA) in an EI mode of $70 \mathrm{eV}$. The carrier gas, helium, was applied at a flow rate of $1.0 \mathrm{ml} / \mathrm{min}$. The column oven was held at $50{ }^{\circ} \mathrm{C}$ for 5 min before being raised to $290{ }^{\circ} \mathrm{C}$ at the rate of $10^{\circ} \mathrm{C} / \mathrm{min}$ and then held constant for $5 \mathrm{~min}$. The molecular separator temperature and ion source temperature were 250 and $260{ }^{\circ} \mathrm{C}$, respectively. The derivatized samples $(1 \mu \mathrm{l})$ were injected into the GC-MS system in the splitless mode.

\section{Results and Discussion}

To answer the questions listed in the introduction, the reaction between 13-HPODE and DCBQ (used as a model halogenated quinone) was studied first, and their radical intermediates and final products were identified by the complementary application of ESR spin trapping, HPLC-MS, GC-MS, and various other analytical methods. In comparison, the classic Fe(II)-mediated decomposition of 13-HPODE was also investigated in parallel.

DCBQ could enhance the decomposition of 13-HPODE and formation of lipid alkyl radical intermediates

It has been shown that $\mathrm{Fe}(\mathrm{II})$ can enhance the decomposition of 13-HPODE to form various lipid-derived radicals such as lipid alkoxyl radical, lipid peroxyl radical, and carbon-centered lipid alkyl radicals [7]. The most widely accepted method for detecting and identifying unstable radical species is the ESR spin trapping method. The nitrone compound POBN has been frequently used as a spin trapping agent to detect a variety of lipid-derived carboncentered radicals. We found that, in the presence of POBN, the typical POBN/carbon-centered radical adducts with the characteristic six-line ESR signal could be produced only when DCBQ was combined together with 13-HPODE (Fig. 1C), and not by either of them alone. Similar POBN radical adducts were also observed during $\mathrm{Fe}(\mathrm{II})$-mediated decomposition of 13-HPODE (Fig. 1D), which is in agreement with previous studies [6,7]. These results indicate that DCBQ can enhance the decomposition of 13-HPODE and formation of the carbon-centered radical intermediates. However, it is well known that various POBN lipid alkyl radical adducts showed almost identical six-line ESR spectra, which may actually contain several different carbon-centered radical adducts. To distinguish different lipid radicals produced during DCBQ-
A

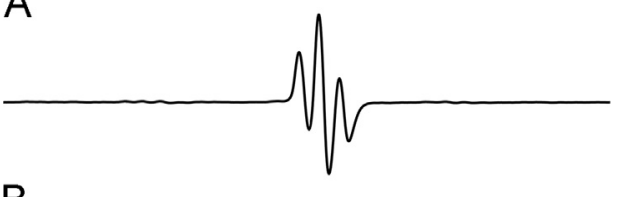

B

C

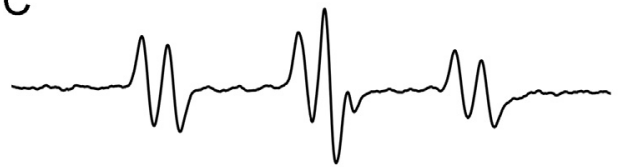

$\mathrm{D}$

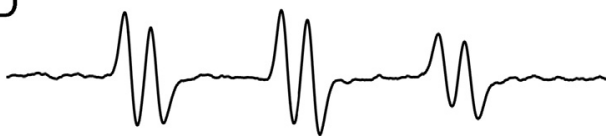

Fig. 1. ESR spectra of POBN radical adducts produced by $13-\mathrm{HPODE}$ and 2,5-DCBQ or $\mathrm{Fe}(\mathrm{II})$. Reactions were carried out at room temperature in Chelex-treated phosphate buffer ( $100 \mathrm{mM}, \mathrm{pH} 7.4)$. All reaction mixtures contained $20 \mathrm{mM}$ POBN. (A) $0.5 \mathrm{mM}$ DCBQ (B) $3 \mathrm{mM} 13-\mathrm{HPODE}$, (C) $0.5 \mathrm{mM}$ DCBQ + $3 \mathrm{mM} 13-\mathrm{HPODE}$, (D) $0.5 \mathrm{mM} \mathrm{Fe}(\mathrm{II})+3 \mathrm{mM}$ 13-HPODE. ESR spectra were recorded $1 \mathrm{~min}$ after the interaction between reactants at room temperature under normal room-lighting conditions. The central signal in the spectrum for DCBQ alone was identified as the 2,5 -dichlorosemiquinone anion radical $\left(\mathrm{DCSQ}^{-{ }^{-}}\right)\left(\alpha^{\mathrm{H}}=2.02 \mathrm{G} ; g=2.0050\right)$. The hyperfine splitting constants for the POBN/carbon-centered radical adduct were $\alpha^{\mathrm{H}}=2.5 \mathrm{G}$ and $\alpha^{\mathrm{N}}=15.7 \mathrm{G}$.

mediated decomposition of 13-HPODE, we have combined HPLC with mass spectrometry, by which different POBN adducts with similar six-line spectra could be separated and then identified. Because of the low levels of spin trapped radical products in the reaction mixture, the SRM mode of the calculated protonated molecule mass $( \pm 0.5 \mathrm{Da})$ of predicted products was used for HPLC-MS and MS/MS analysis. In the DCBQ/13-HPODE/POBN system, the following POBN/radical adducts were detected using the above method: (1) POBN/pentyl radical adduct (Fig. 2IA), with the SRM transition at $m / z 266 / 179$ (Fig. 2IB), where the ion at $m / z$ 266 corresponds to the protonated molecule of POBN/pentyl radical adduct $[\mathrm{M}+\mathrm{H}]^{+*}$, and the fragment ion at $\mathrm{m} / z 179$ corresponds to the loss of $\left(\mathrm{CH}_{3}\right)_{3} \mathrm{C}(\mathrm{O}) \mathrm{N}$ from the protonated molecule (Fig. 2IC); (2) POBN/7-carboxyheptyl radical adduct (Fig. 2IIA), with the SRM transitions at $m / z$ 338/251 (Fig. 2IIB), where the ion $\mathrm{m} / \mathrm{z} 338$ corresponds to the protonated molecule of POBN/7carboxyheptyl radical adduct $[\mathrm{M}+\mathrm{H}]^{+}$, and the fragment ion at $\mathrm{m} / \mathrm{z} 251$ corresponds to the loss of $\left(\mathrm{CH}_{3}\right)_{3} \mathrm{C}(\mathrm{O}) \mathrm{N}$ from the protonated molecule (Fig. 2IIC); (3) other POBN/radical adducts with the same ion peak at $m / z 490$, which was considered to be either 1-(7carboxyheptyl)-4,5-epoxy-2-decenyl or 1-(1,2-epoxyheptyl)-10carboxy-2-decenyl radical adduct or their corresponding isomers (Fig. 2III). Tandem MS analysis ( $m / z 266$ and $m / z$ 338) was consistent with the proposed structure (Fig. 2IC and IIC).

In previous studies, lipid alkoxyl radicals were also found to be produced during Fe(II)-mediated decomposition of 13-HPODE [6]; and in our recent study, a carbon-centered quinone ketoxy radical, - $\mathrm{CBQ}=\mathrm{O}$, the spin isomer of the oxygen-centered enoxy radical $\mathrm{CBQ}^{-} \mathrm{O}^{-}$, was detected and identified during $\mathrm{DCBQ}-$ mediated decomposition of $t-\mathrm{BuOOH}$ [10]. However, during the reaction of DCBQ and 13-HPODE, we could not directly detect either of these two radicals with POBN or DMPO as the spin trapping agent. The spin trapping of secondary lipid alkoxyl radicals in aqueous systems has been proven difficult, apparently because of their rapid cyclization [17]; whereas the steady-state concentration of - $\mathrm{CBQ}=\mathrm{O}$ might be too low to be detected by ESR spin trapping in this system. 

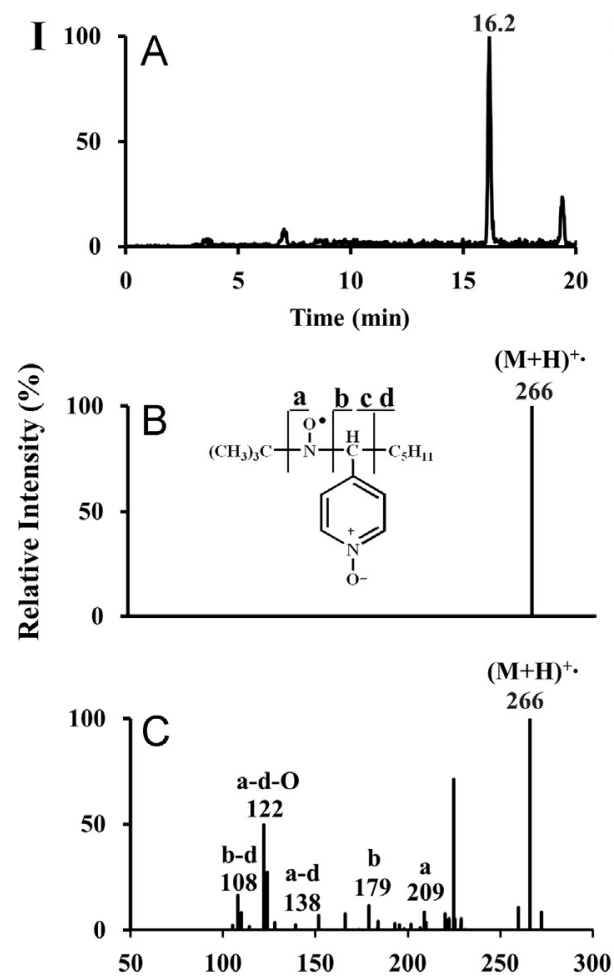
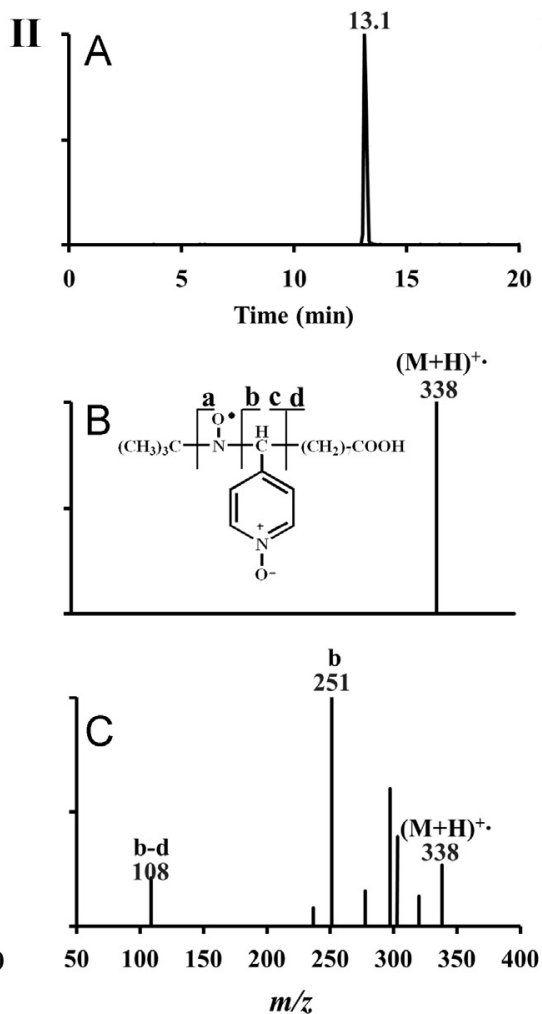
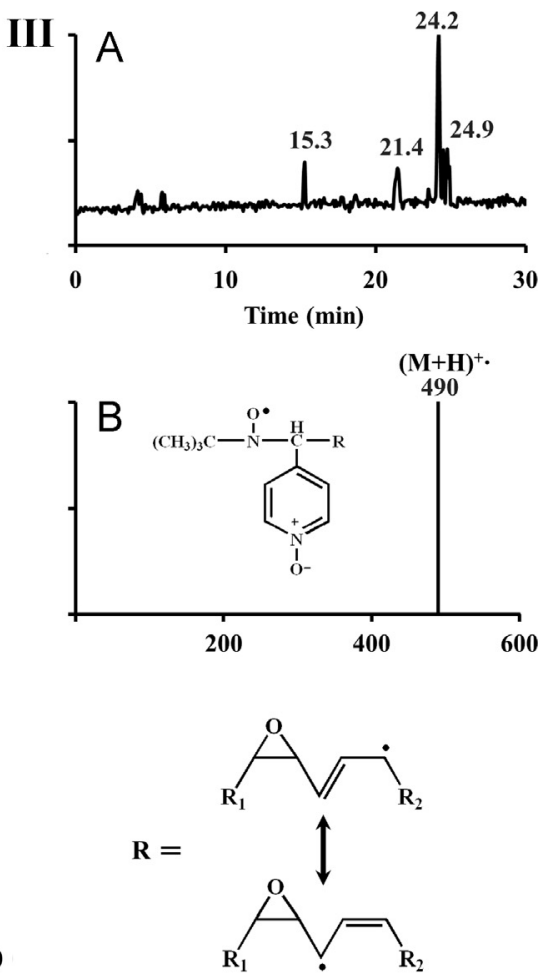

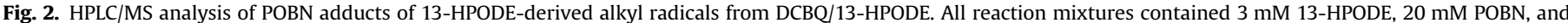

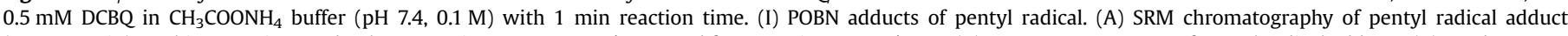

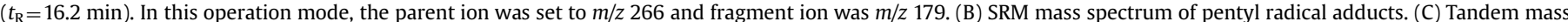

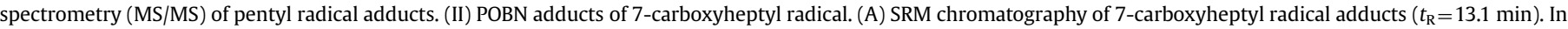

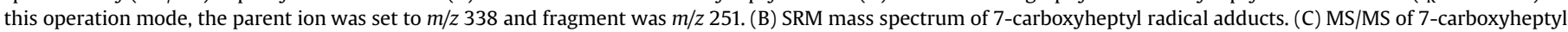

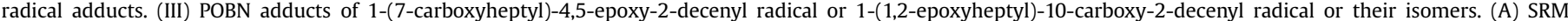

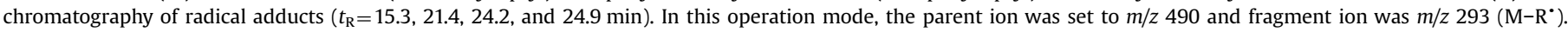
(B) SRM mass spectrum of radical adducts. Because of the low levels of these spin trapped radical products, we could not get their MS/MS.

The detection of the two quinone-lipid alkoxyl conjugates and $\mathrm{CBQ}-\mathrm{OH}$, however, as shown below, may provide indirect evidence for the formation of these two radicals.

Two quinone-lipid alkoxyl conjugates were identified as the major reaction products between $D C B Q$ and $13-H P O D E$

In our recent study, two major reaction products between DCBQ and $t-\mathrm{BuOOH}$ were identified as $\mathrm{CBQ}(\mathrm{OH})-\mathrm{O}-t-\mathrm{Bu}$ and $\mathrm{CBQ}-\mathrm{OH}[11]$. We speculated that analogous reaction products might be formed between DCBQ and 13-HPODE. As expected, $\mathrm{CBQ}-\mathrm{OH}$ was readily identified as one of the reaction products, which showed the same retention time and MS profile compared with the authentic $\mathrm{CBQ}-\mathrm{OH}$ (Fig. 3A).

A trace amount of 2-hydroxy-3-(L-13-oxy)-5-chloro-1,4-benzoquinone $(\mathrm{CBQ}(\mathrm{OH})-13-\mathrm{O}-\mathrm{L}$; for simplicity, here we refer to $13-$ HPODE as 13-LOOH) was detected by HPLC-MS with ion peak clusters at $m / z 451$ (Fig. 3B). Fourier transform ion cyclotron resonance (FTICR) mass spectrometry is one of the techniques that can provide high mass accuracy and high mass resolution. To get a more accurate molecular weight, elemental composition, and structural information for $\mathrm{CBQ}(\mathrm{OH})-13-\mathrm{O}-\mathrm{L}$, FTICR-MS was used for further studies. $\mathrm{CBQ}(\mathrm{OH})-13-\mathrm{O}-\mathrm{L}$ was characterized by FTICRMS with one-chlorine isotope peak clusters at $m / z$ 451.18942, which corresponds well to its deprotonated molecule with theoretical value at 451.18929 (Supplementary Table S1).

Interestingly and unexpectedly, another new quinone-O-L reaction product with ion peak clusters at $\mathrm{m} / \mathrm{z} 467$ was also observed (Fig. 3C). FTICR-MS showed that this product is probably 2-hydroxy-3-(OL-9-oxy)-5-chloro-1,4-benzoquinone ( $\mathrm{CBQ}(\mathrm{OH})-9$ -
O-LO), which was characterized with one-chlorine isotope peak clusters at $m / z 467.18388$ and corresponds well to its deprotonated molecule with a theoretical value at 467.18420 . It is known that a new lipid hydroperoxide, 12,13-epoxy-9-hydroperoxy-10-octadecenoic acid $(9-\mathrm{OLOOH})$, might be formed during the process of 13-HPODE decomposition (see Supplementary Scheme S2), which may further react with $D C B Q$ to generate this new reaction product.

These results indicate that a nucleophilic reaction may take place between DCBQ and 13-HPODE, first forming a quinone peroxide (CBQ-13-OOL) intermediate, which can decompose homolytically to produce the lipid alkoxyl radical $13-\mathrm{LO}^{\circ}$ and $\mathrm{CBQ}^{\circ} \mathrm{O}^{\circ}$. $\mathrm{CBQ}-\mathrm{O}^{\circ}$ may isomerize to form the carbon-centered quinone ketoxy radical ${ }^{\circ} \mathrm{CBQ}=\mathrm{O}$, which could then couple with $13-\mathrm{LO}^{*}$ to produce one of the major reaction products, $\mathrm{CBQ}(\mathrm{OH})$ 13-O-L (see Scheme 1).

\section{Identification of several long-chain lipid acids derived from 13-HPODE}

The lipid alkoxyl radical $13-\mathrm{LO}^{\bullet}$ is a relatively reactive radical, so not only will it react with quinone ketoxy radical to form conjugates, but the transfer of radical center and cleaving of adjacent $\mathrm{C}-\mathrm{C}$ bonds may also lead to the generation of various long-chain lipid acids (Supplementary Scheme S2). Four ion peaks at $\mathrm{m} / \mathrm{z} 227, \mathrm{~m} / \mathrm{z} 329, \mathrm{~m} / \mathrm{z} 293$, and $\mathrm{m} / \mathrm{z} 311$ (Figs. 4IB and 4II) were observed by HPLC-MS from the reaction of 13-HPODE and Fe(II). Based on FTICR-MS data (Supplementary Table S1) and previous studies $[7,8]$, all of these compounds could be identified. They are 11-formyl-11-hydroxy-9-undecenoic acid (compound I), 12,13-epoxy-9-hydroperoxy-10-octadecenoic acid (compound II), 


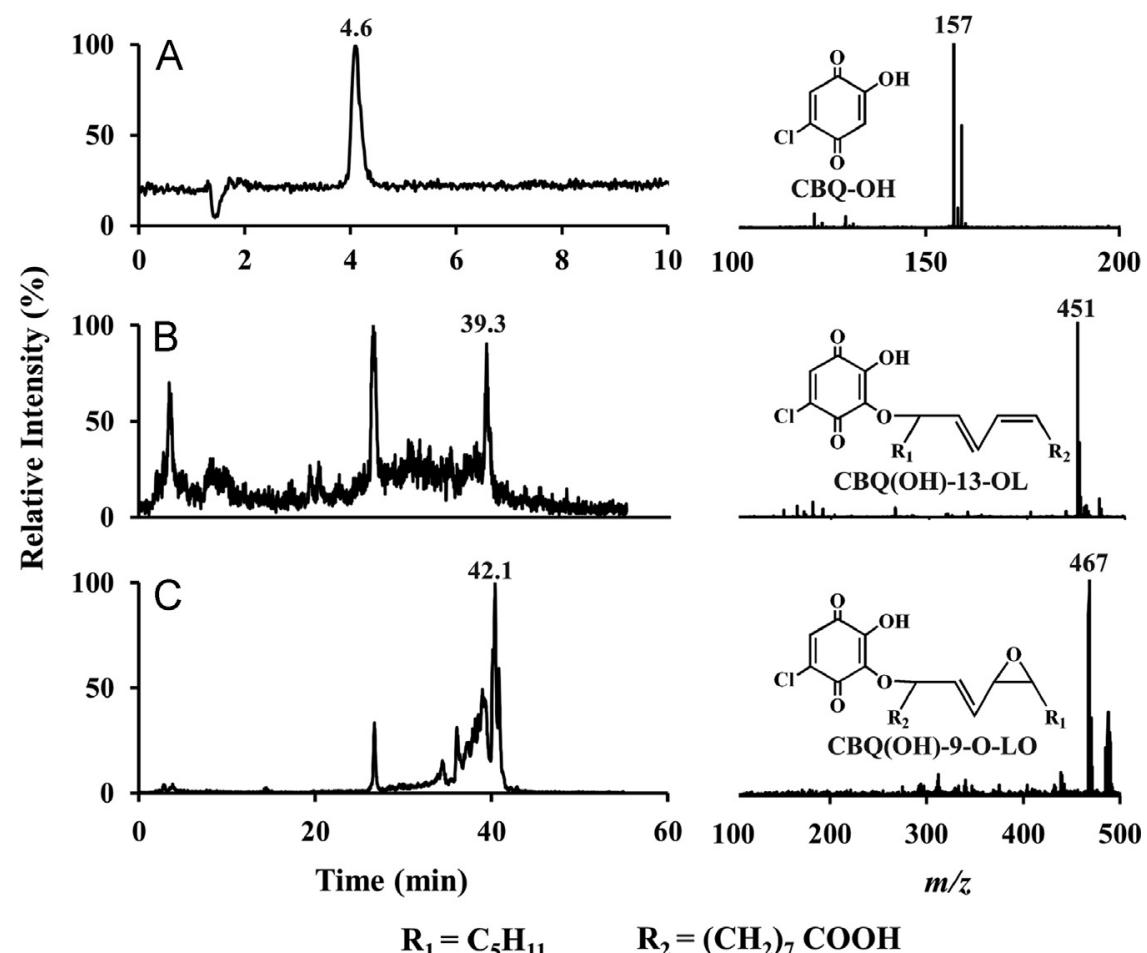

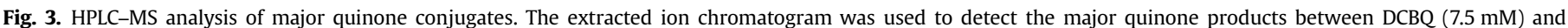

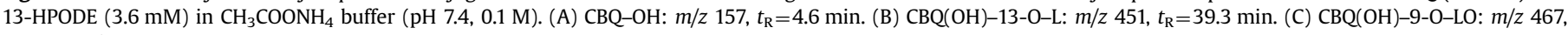
$t_{\mathrm{R}}=42.1 \mathrm{~min}$
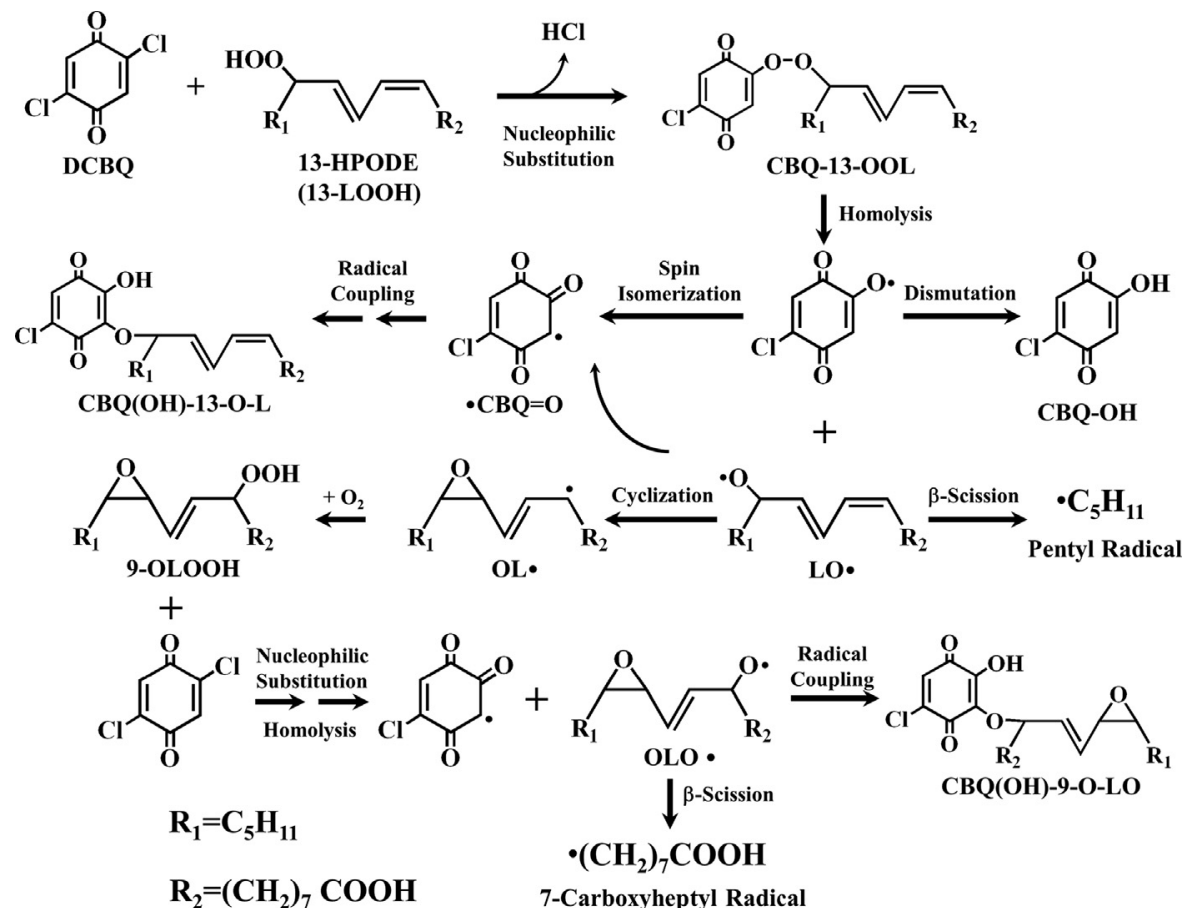

Scheme 1. Proposed molecular mechanism for metal-independent decomposition of 13-HPODE by DCBQ.

13-oxo-9,11-octadecadienoic acid (compound III), and 12,13epoxy-11-hydroxy-9-octadecenoic acid (compound IV). When Fe (II) was substituted by DCBQ in addition to compounds I-IV, a new ion peak at $m / z$ 295, which corresponds to 13-hydroxy-9,11octadecadienoic acid (compound V), was also observed (Figs. 4IA and II).
DCBQ could enhance the decomposition of 13-HPODE and formation of the genotoxic aldehyde 4-hydroxy-2-nonenal

Because $\mathrm{Fe}(\mathrm{II})$ has been shown to enhance the formation of HNE from the decomposition of 13-HPODE (Supplementary Scheme S3) $[9,18,19]$, we speculated that DCBQ may also enhance 

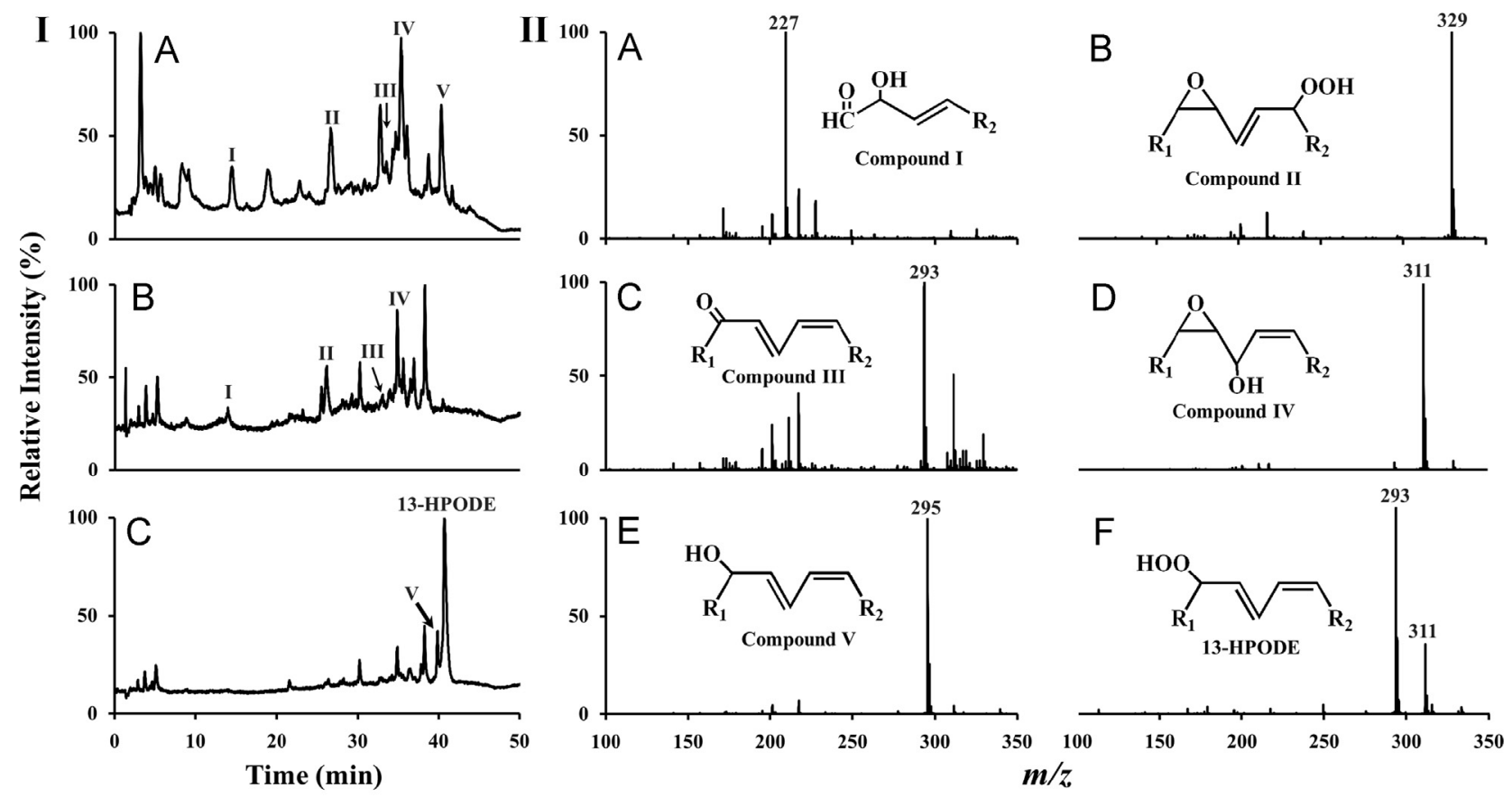

$$
\mathbf{R}_{1}=\mathrm{C}_{5} \mathbf{H}_{11} \quad \mathbf{R}_{2}=\left(\mathrm{CH}_{2}\right)_{7} \mathbf{C O O H}
$$

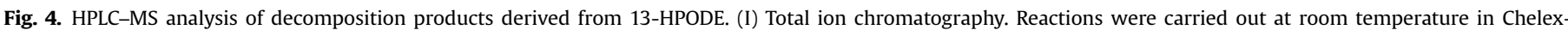

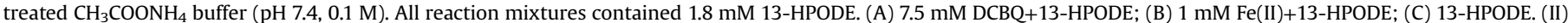

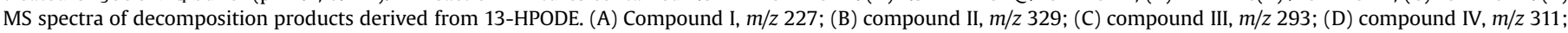
(E) compound V, $m / z$ 295; (F) 13-HPODE.
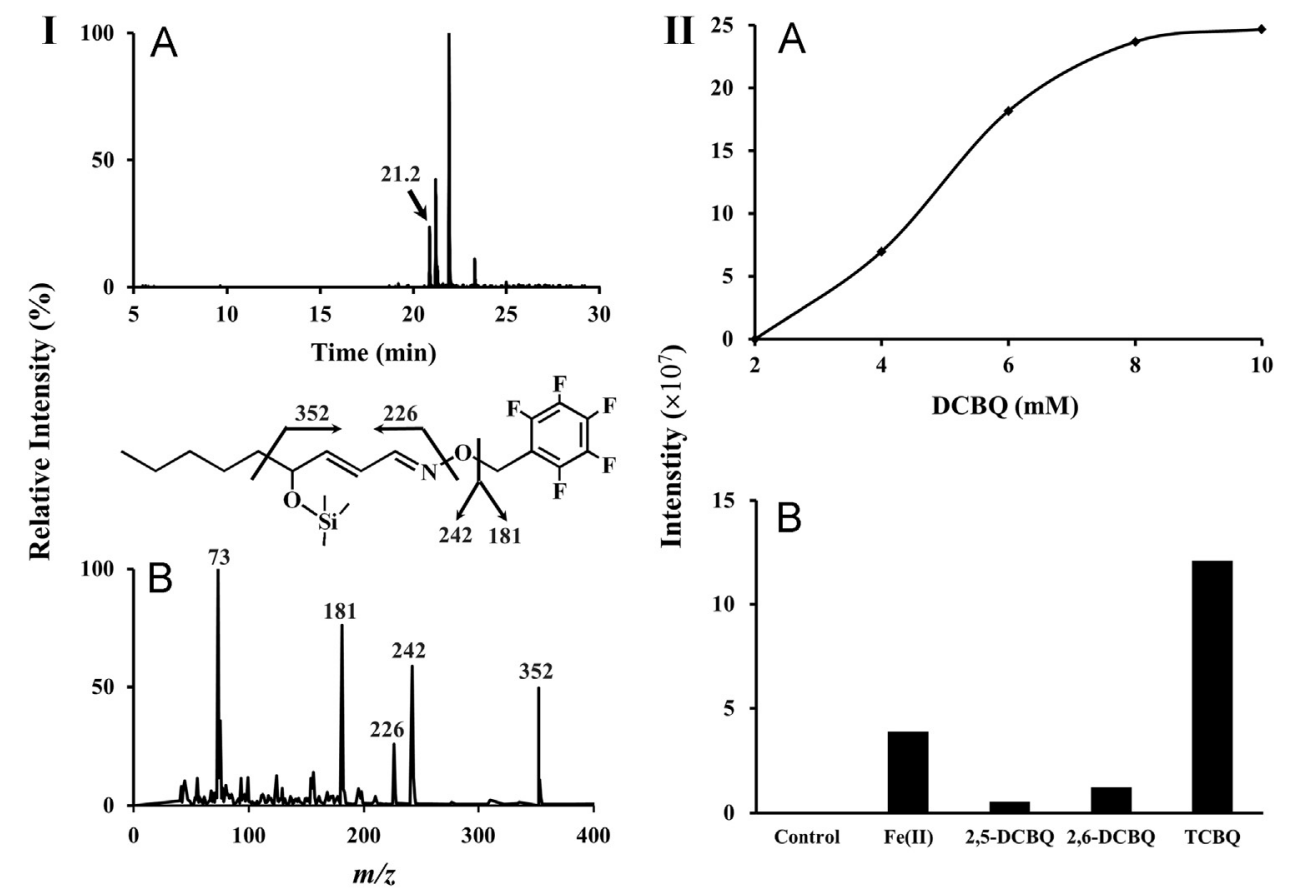

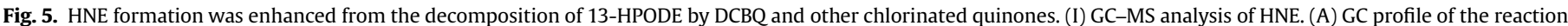

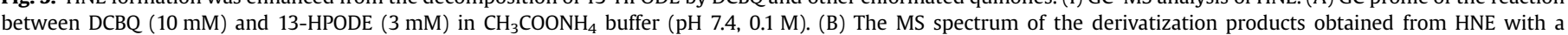

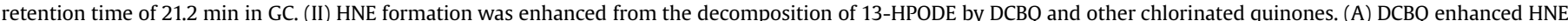

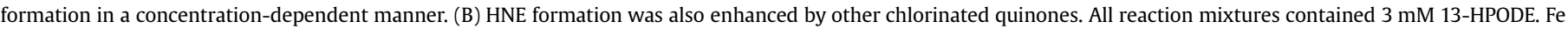
(II), $1 \mathrm{mM}$; 2,5-DCBQ, $10 \mathrm{mM}$; 2,6-DCBQ $10 \mathrm{mM}$; TCBQ $10 \mathrm{mM}$.

HNE formation. To test whether this is the case, HNE was measured by GC-MS after derivatization (Fig. 5I). The aldehyde groups were derivatized into their PFB-oximes, which universally exhibited a fragment ion at $m / z 181$, and the hydroxyl groups were further derivatized into their TMS derivatives. These derivatizations give the characteristic fragment ions of aldehydes and help us to identify HNE in the GC-MS [20]. We found that DCBQ could indeed enhance the decomposition of 13-HPODE and formation of HNE in a concentration-dependent manner (Fig. 5IIA). Similar effects were observed when DCBQ was 
Table 1

The comparison of major differences and similarities for the three reaction systems.

\begin{tabular}{|c|c|c|c|c|c|}
\hline Reaction & Alkoxyl radical & C-centered radicals & Hydroxylated quinone & Quinone conjugates & Lipid acids and HNE \\
\hline DCBQ/13-HPODE & $\mathrm{LO}^{*}$ & $\begin{array}{l}\cdot{ }^{-} \mathrm{C}_{5} \mathrm{H}_{11} \\
\cdot\left(\mathrm{CH}_{2}\right)_{7} \mathrm{COOH} \\
\mathrm{OL}^{\cdot} \\
\cdot{ }^{\mathrm{CBQ}}=\mathrm{O}\end{array}$ & $\mathrm{CBQ}-\mathrm{OH}$ & $\begin{array}{l}\mathrm{CBQ}(\mathrm{OH})-13-\mathrm{O}-\mathrm{L} \\
\mathrm{CBQ}(\mathrm{OH})-9-\mathrm{O}-\mathrm{LO}\end{array}$ & Compounds I, II, III, IV, and V; HNE \\
\hline $\mathrm{Fe}(\mathrm{II}) / 13-\mathrm{HPODE}$ & $\mathrm{LO}^{*}$ & $\begin{array}{l}\cdot{ }^{-} \mathrm{C}_{5} \mathrm{H}_{11} \\
\cdot\left(\mathrm{CH}_{2}\right)_{7} \mathrm{COOH} \\
\mathrm{OL}^{\cdot}\end{array}$ & $\mathrm{N} / \mathrm{A}$ & $\mathrm{N} / \mathrm{A}$ & Compounds I, II, III, IV, and V; HNE \\
\hline $\mathrm{DCBQ} / t-\mathrm{BuOOH}$ & $t-\mathrm{BuO}^{\bullet}$ & $\begin{array}{l}\cdot \mathrm{CH}_{3} \\
\cdot \mathrm{CBQ}=\mathrm{O}\end{array}$ & $\mathrm{CBQ}-\mathrm{OH}$ & $\mathrm{CBQ}(\mathrm{OH})-\mathrm{O}-t-\mathrm{Bu}$ & $\mathrm{N} / \mathrm{A}$ \\
\hline
\end{tabular}

substituted by other chlorinated quinones such as 2,6-DCBQ and TCBQ, which is one of the major genotoxic quinoid metabolites of the widely used wood preservative pentachlorophenol (Fig. 5IIB). It is worth noting that TCBQ was more effective than DCBQ at enhancing HNE formation from 13-HPODE.

\section{Molecular mechanism for metal-independent decomposition of $13-H P O D E$ by DCBQ}

The detection and identification of the transient lipid-derived alkyl radicals, together with the identification of the major reaction products $\mathrm{CBQ}-\mathrm{OH}$ and two quinone-O-L conjugates, strongly support the following mechanism for DCBQ-mediated decomposition of 13-HPODE (Scheme 1): a nucleophilic reaction may take place between DCBQ and 13-HPODE (13-LOOH), forming a quinoneperoxide intermediate $\mathrm{CBQ}-13-\mathrm{OOL}$, which may decompose homolytically to produce the lipid alkoxyl radical $13-\mathrm{LO}^{*}$ and the oxygen-centered quinone enoxy radical (CBQ- $\left.\mathrm{O}^{\circ}\right)$. CBQ- $\mathrm{O}^{*}$ then either disproportionates to form one of the major reaction products, $\mathrm{CBQ}-\mathrm{OH}$, or isomerizes to form the carbon-centered quinone ketoxy radical ${ }^{\circ} \mathrm{CBQ}=\mathrm{O}$, which then couples with $13-\mathrm{LO}^{\circ}$ to produce another major reaction product, $\mathrm{CBQ}(\mathrm{OH})-13-\mathrm{O}-\mathrm{L}$, via keto-enol tautomerization. The $13-\mathrm{LO}^{*}$ could either lead to the formation of pentyl radical via $\beta$-scission or isomerize to form the carbon-centered radical $\mathrm{OL}^{\circ}$, which then adds oxygen to form a new lipid hydroperoxide, 9-OLOOH. 9-OLOOH then may react with DCBQ to generate a new alkoxyl radical, 9-OLO', which either couples with ${ }^{\circ} \mathrm{CBQ}=\mathrm{O}$ to produce the new conjugate product $\mathrm{CBQ}(\mathrm{OH})-9-\mathrm{O}-\mathrm{LO}$ or, via $\beta$-scission, leads to the formation of 7-carboxyheptyl radical. The mechanism of the enhanced formation of HNE by DCBQ might be similar to that by $\mathrm{Fe}(\mathrm{II})$ (Supplementary Scheme S3).

What are the unique differences and similarities for the reactions in this study?

In general, the reaction mechanisms of DCBQ with $t-\mathrm{BuOOH}$ and 13-HPODE are very similar; both are through nucleophilic substitution and homolytical decomposition (Scheme 1 and 2 in Ref. [7]). The major differences between them are the specific reaction radical intermediates and products. Because 13-HPODE is a long-chain secondary hydroperoxide, its decomposition is much more complicated, which results in more radical intermediates and lipid acids (summarized in Table 1).

The major difference between the reactions of DCBQ and Fe(II) with 13-HPODE is the reaction mechanism: whereas $\mathrm{Fe}(\mathrm{II})-$ mediated decomposition is via a Fenton-like redox reaction, DCBQ-mediated decomposition is through nucleophilic substitution and homolytical decomposition. Although their reaction mechanisms are different, the major reaction radical intermediates and products are very similar, especially those derived from the lipid alkoxyl radical 13-LO (Table 1).

\section{Potential biological implications}

Our observation that not only DCBQ but also other chlorinated quinones can react with 13-HPODE to produce the reactive lipid alkyl radicals and the genotoxic HNE in a metal-independent manner has interesting biological implications. For example, this may partly explain the potential carcinogenicity of the widely used wood preservative pentachlorophenol (PCP), which has been listed as a priority pollutant by the U.S. EPA and classified as a group $2 \mathrm{~B}$ environmental carcinogen [16], and other widely used polyhalogenated aromatic compounds such as 2,4,6- and 2,4,5-trichlorophenol, hexachlorobenzene, Agent Orange, and the brominated flame retardant 3,3',5,5'-tetrabromobisphenol A. It has been shown that these compounds can be metabolized in vivo [16,21-25], or dehalogenated chemically and enzymatically [26-28], to their corresponding quinones. Recently, several polychlorinated quinoid compounds, such as 2,6-DCBQ which were suspected bladder carcinogens, were also identified as new chlorination disinfection by-products in drinking water [29] and found in discharges from pulp and paper mills [16].

The $\alpha, \beta$-unsaturated aldehydes are highly reactive and readily react with proteins, DNA, and phospholipids, by Michael addition reaction and/or a Schiff base formation, to cause deleterious effects. Among all aldehydes detected, which varied in chain length from 8 to 11 carbons, the 9-carbon HNE was the most prominent and has been most extensively studied because of its high bioactivity. HNE was recognized as having the greatest toxicological potential among these aldehyde products and over the years has become a paradigm for studying the pathophysiological effects of the cytotoxic products of LPO. HNE-protein adducts are considered to be good biomarkers of LPO in vivo [30]. HNE is thought to play a central role in various traumatic injuries and disease states that involve cellular oxidative stress, for example, spinal cord trauma, diabetes, and Alzheimer disease [1-5]. It should be noted that aldehydes are not only toxic end products and remnants of LPO but also may act as signaling messengers [31]. According our findings, halogenated quinones may play important roles in LPO, because they could enhance the decomposition of 13-HPODE to form reactive lipid-derived radicals and genotoxic aldehyde HNE. Because 13-HPODE is an endogenous lipid hydroperoxide, the results from this study should be more physiologically relevant compared with our previous studies with $t$-BuOOH and halogenated quinones. Our new findings may provide a new perspective to better understand the genotoxicity and carcinogenicity of PCP and other polyhalogenated aromatic compounds. Further studies are needed to investigate whether this is true in animal model systems.

\section{Acknowledgments}

The work in this paper was supported by Project 973 (2008CB418106); the Hundred-Talent Project, CAS; an NSF China 
Outstanding Youth Award (20925724); NSFC Grants 21237005, 21207150, 21207139, 21077058, 21321004 and 20921063; and NIH Grants ES11497, RR01008, and ES00210. We thank Drs. Xin-Ying Zhao and Xin-Lei Gou for their technical help.

\section{Appendix A. Supporting information}

Supplementary data associated with this article can be found in the online version at http://dx.doi.org/10.1016/j.freeradbiomed. 2013.05.008.

\section{References}

[1] Yin, H.; Xu, L.; Porter, N. A. Free radical lipid peroxidation: mechanisms and analysis. Chem. Rev. 111:5944-5972; 2011.

[2] Niki, E. Lipid peroxidation: physiological levels and dual biological effects. Free Radic. Biol. Med. 47:469-484; 2009.

[3] Halliwell, B.; Gutteridge, J. Free Radicals in Biology and Medicine. London: Oxford Univ. Press; 2007.

[4] Blair, I. A. DNA adducts with lipid peroxidation products. J. Biol. Chem. 283:15545-15549; 2008

[5] Lee, S. H.; Oe, T.; Blair, I. A. Vitamin C-induced decomposition of lipid hydroperoxides to endogenous genotoxins. Science 292:2083-2086; 2001.

[6] Qian, S. Y.; Yue, G. H.; Tomer, K. B.; Mason, R. P. Identification of all classes of spin trapped carbon-centered radicals in soybean lipoxygenase-dependent lipid peroxidations of $\omega-6$ polyunsaturated fatty acids via LC/ESR, LC/MS, and tandem MS. Free Radic. Biol. Med 34:1017-1028; 2003.

[7] Iwahashi, H.; Hirai, T. Kumamoto, K. High performance liquid chromatography/electron spin resonance/mass spectrometry analyses of radicals formed in an anaerobic reaction of 9- (or 13-) hydroperoxide octadecadienoic acids with ferrous ions. J. Chromatogr. A 1132:67-75; 2006.

[8] Spiteller, P.; Kern, W.; Reiner, J.; Spiteller, G. Aldehydic lipid peroxidation products derived from linoleic acid. Biochim. Biophys. Acta 1531:188-208; 2001.

[9] Schneider, C.; Porter, N. A.; Brash, A. R. Routes to 4-hydroxynonenal: fundamental issues in the mechanisms of lipid peroxidation. J. Biol. Chem. 283:15539-15543; 2008.

[10] Zhu, B. Z.; Zhao, H. T.; Kalyanaraman, B.; Liu, J.; Shan, G. Q.; Du, Y. G.; Frei, B. Mechanism of metal-independent decomposition of organic hydroperoxides and formation of alkoxyl radicals by halogenated quinones. Proc. Natl. Acad. Sci. USA 104:3698-3702; 2007.

[11] Zhu, B. Z.; Shan, G. Q.; Huang, C. H.; Kalyanaraman, B.; Mao, L.; Du, Y. G. Metalindependent decomposition of hydroperoxides by halogenated quinones: detection and identification of a quinone ketoxy radical. Proc. Natl. Acad. Sci. USA 106:11466-11471; 2009.

[12] Zhu, B. Z.; Zhao, H. T.; Kalyanaraman, B.; Frei, B. Metal-independent production of hydroxyl radicals by halogenated quinones and hydrogen peroxide: an ESR spin trapping study. Free Radic. Biol. Med. 32:465-473; 2002.
[13] Zhu, B. Z.; Kalyanaraman, B.; Jiang, G. B. Molecular mechanism for metalindependent production of hydroxyl radicals by hydrogen peroxide and halogenated quinones. Proc. Natl. Acad. Sci. USA 104:17575-17578; 2007.

[14] Zhu, B. Z.; Zhu, J. G.; Mao, L.; Kalyanaraman, B.; Shan, G. Q. Detoxifying carcinogenic polyhalogenated quinones by hydroxamic acids via an unusual double Lossen rearrangement mechanism. Proc. Natl. Acad. Sci. USA 107:20686-20690; 2010.

[15] Zhu, B. Z.; Mao, L.; Huang, C. H.; Qin, H.; Fan, R. M.; Kalyanaraman, B.; Zhu, J. G. Unprecedented hydroxyl radical-dependent two-step chemiluminescence production by polyhalogenated quinoid carcinogens and $\mathrm{H}_{2} \mathrm{O}_{2}$. Proc. Natl. Acad. Sci. USA 109:16046-16051; 2012.

[16] Zhu, B. Z.; Shan, G. Q. Potential mechanism for pentachlorophenol-induced carcinogenicity: a novel mechanism for metal-independent production of hydroxyl radicals. Chem. Res. Toxicol. 22:969-977; 2009.

[17] Grossi, L.; Strazzari, S.; Gilbert, B. C.; Whitwood, A. C. Oxiranylcarbinyl radicals from allyloxyl radical cyclization: characterization and kinetic information via ESR spectroscopy. J. Org. Chem. 63:8366-8372; 1998.

[18] Lee, S. H.; Blair, I. A. Characterization of 4-oxo-2-nonenal as a novel product of lipid peroxidation. Chem. Res. Toxicol. 13:698-702; 2000.

[19] Lee, S. H.; Oe, T.; Arora, J. S.; Blair, I. A. Analysis of FelI-mediated decomposition of a linoleic acid-derived lipid hydroperoxide by liquid chromatography/ mass spectrometry. J. Mass Spectrom. 40:661-668; 2005.

[20] Kawai, Y.; Takeda, S.; Terao, J. Lipidomic analysis for lipid peroxidation-derived aldehydes using gas chromatography-mass spectrometry. Chem. Res. Toxicol. 20:99-107; 2007.

[21] Bolton, J. L.; Trush, M. A.; Penning, T. M.; Dryhurst, G.; Monks, T. J. Role of quinones in toxicology. Chem. Res. Toxicol. 13:135-160; 2000.

[22] Song, Y.; Wagner, B. A.; Witmer, J. R.; Lehmler, H. J.; Buettner, G. R. Nonenzymatic displacement of chlorine and formation of free radicals upon the reaction of glutathione with PCB quinones. Proc. Natl. Acad. Sci. USA 106:9725-9730; 2009.

[23] Chignell, C. F.; Han, S. K.; Mouithys-Mickalad, A.; Sik, R. H.; Stadler, K. Kadiiska, M. B. EPR studies of in vivo radical production by 3,3'5,5'-tetrabromobisphenol A (TBBPA) in the Sprague-Dawley rat. Toxicol. Appl. Pharmacol. 230:17-22; 2008.

[24] Teuten, E. L.; Xu, L.; Reddy, C. M. Two abundant bioaccumulated halogenated compounds are natural products. Science 307:917-920; 2005

[25] Kelly, B. C.; Ikonomou, M. G.; Blair, J. D.; Morin, A. E.; Gobas, F. A. Food webspecific biomagnification of persistent organic pollutants. Science 317:236-239; 2007.

[26] Meunier, B. Catalytic degradation of chlorinated phenols. Science 296:270$271 ; 2002$.

[27] Gupta, S. S.; Stadler, M.; Noser, C. A.; Ghosh, A.; Steinhoff, B.; Lenoir, D.; Horwitz, C. P.; Schramm, K. W.; Collins, T. J. Rapid total destruction of chlorophenols by activated hydrogen peroxide. Science 296:326-328; 2002.

[28] Sorokin, A.; Meunier, B.; Seris, J. L. Efficient oxidative dechlorination and aromatic ring cleavage of chlorinated phenols catalyzed by iron sulfophthalocyanine. Science 268:1163-1166; 1995.

[29] Zhao, Y.; Qin, F.; Boyd, J. M.; Anichina, J.; Li, X. F. Characterization and determination of chloro- and bromo-benzoquinones as new chlorination disinfection byproducts in drinking water. Anal. Chem. 82:4599-4605; 2010.

[30] Petersen, D. R.; Doorn, J. A. Reactions of 4-hydroxynonenal with proteins and cellular targets. Free Radic. Biol. Med. 37:937-945; 2004.

[31] Awasthi, Y. C.; Sharma, R.; Sharma, A.; Yadav, S.; Singhal, S. S.; Chaudhary, P.; Awasthi, S. Self-regulatory role of 4-hydroxynonenal in signaling for stressinduced programmed cell death. Free Radic. Biol. Med. 45:111-118; 2008. 